4

\title{
How well do we know the Circumference of a Storage Ring?
}

\author{
M. Beckmann, DESY \\ V. Ziemann, Uppsala University
}

\section{Introduction}

For precision spectroscopical measurements of narrow states in a storage ring such as HESR [1] in the FAIR facility at GSI [2] the absolute energy of the stored beam needs to be known to a relative accuracy in the $10^{-5}$ range. The prime experiment in the HESR is the PANDA [3] detector with an extensive physics program with Hadron spectroscopy as one of its main topics. PANDA will search for gluonic excitations such as glueballs or hybrids as well as well as perform detailed scans of the spectrum of charmonium systems. A third activity will address the spectrum and decay width of the recently discovered D-mesons. For these experiments the ring will operate close to the production threshold of the mesons which therefore requires precise knowledge of the absolute value of the beam energy. When operating in an energy range where an electron cooler is available, this accuracy is given by the precision to which the acceleration voltage and therefore the velocity of the electrons is known. When operating at higher energies, exceeding the energy range of the cooler, one has to rely on a precise knowledge of the frequency of the radio-frequency $(\mathrm{RF})$ system for the revolution time and the knowledge of the circumference of the storage ring to determine the velocity and thereby the energy of the stored ion beam. At first sight the circumference appears to be a trivially well-known quantity, but when requiring accuracies in the $10^{-5}$ range, which means $5 \mathrm{~mm}$ for a $500 \mathrm{~m}$ ring, this is not entirely self-evident. In fact, production measurements of mesons at threshold in CELSIUS revealed a discrepancy of $47 \mathrm{~mm}$ in the $82 \mathrm{~m}$ circumference of CELSIUS [4] as designed and as determined by the experimental group [5]. The memory of this discrepancy triggered our investigation even though CELSIUS is long dismantled.

The origins of the lack of knowledge about the circumference lie in the finite precision of the surveying procedure that is used to place magnets at their design positions. The primary source are the dipoles which define the reference orbit, but also transversely misaligned quadrupoles which will cause horizontal oscillations of 
the closed orbit. If the closed orbit lies further towards the outside in a dipole magnet, the length of the orbit is increased. Note that the dominant source of orbit length variation happens in the bending plane which is normally horizontal. A related source are other vertical magnetic stray fields that cause horizontal oscillations, similar to those caused by misaligned quadrupoles. Of course, these oscillations are corrected by the orbit correction system, which will add more vertical fields, which cause more horizontal oscillations, that are intended to minimize the orbit offset on the beam-position monitors (BPM). The BPMs have a finite resolution which means that the excitations to the orbit correction dipoles has a finite variance which causes the length of the orbit to vary.

We observe that the presence of RF complicates the dynamics further. If magnet misalignments change the length of the orbit, the beam must adjust its energy in order to maintain the revolution time as dictated by the RF system. A change of momentum $\Delta p / p$ and orbit length $\Delta s$ causes a change of revolution time $\Delta T$ according to

$$
\frac{\Delta T}{T}=\left(\alpha-\frac{1}{\gamma^{2}}\right) \frac{\Delta p}{p}+\frac{\Delta s}{C}
$$

where $C$ is the circumference, $T$ the revolution time, $\alpha$ is the momentum compaction factor, and $\gamma$ the beam energy in units of the particle rest mass. Note that the first term on the right hand side describes the commonly used definition of the phase slip factor $\alpha-1 / \gamma^{2}$ where the $\alpha$ describes the change of the orbit length and $1 / \gamma^{2}$ the change of velocity due to the momentum variation. The second term $\Delta s / C$ describes the change of the orbit length and consequently the revolution time due to other independent sources, such as the average bending magnet field strength or an excited steering magnet, an effect we discuss in the next section.

If $\mathrm{RF}$ is present the revolution frequency must remain constant and after changing the orbit length by $\Delta s$ and the beam thus responds by assuming a different momentum $\Delta p / p$ given by

$$
\frac{\Delta p}{p}=-\frac{\Delta s}{\left(\alpha-\frac{1}{\gamma^{2}}\right) C}
$$

which shows that a change in orbit length causes a different energy. It is obvious that the sensitivity of the energy on the orbit length becomes increasingly significant the closer transition is approached.

In the remainder of this report we will assume that all effects that affect the orbit length have a random Gaussian distribution, typically with a RMS of $0.1 \mathrm{~mm}$, which is a typical accuracy achievable by the surveyors. We first discuss the variation of the circumference due to transverse quadrupole misalignment and due to longitudinal 

${ }_{87}$ a location with dispersion $\eta_{0}$. which is given by $[6]$ factor is derived, namely

Inserting $x(s)$ from Eq. 5 we obtain

\section{Orbit length change from a dipole kick}

In this section we calculate the change in the length of the closed orbit due to a horizontal dipole kick. We start by considering the dispersion $\eta_{0}$ at a location $s_{0}$

$$
\eta_{0}=\oint \frac{\sqrt{\beta(s) \beta_{0}}}{2 \sin \pi \nu} \cos \left(\phi_{0}-\phi(s)-\pi \nu\right) \frac{d s}{\rho(s)}
$$

where the integral extends over the entire ring, $\nu$ is the tune, $\rho$ is the radius of curvature of the dipoles, and $\beta_{0}$ and $\phi_{0}$ are the betatron function and phase at position $s_{0}$, respectively. By changing the integration variable from $s$ to $s^{\prime}=s-C$ where $C$ is the circumference of the ring and use that $\rho(s)$ and $\beta(s)$ are periodic functions, but $\phi\left(s^{\prime}\right)$ becomes $\phi(s)-2 \pi \nu$. We then arrive at

$$
\eta_{0}=\oint \frac{\sqrt{\beta\left(s^{\prime}\right) \beta_{0}}}{2 \sin \pi \nu} \cos \left(\phi_{0}-\phi\left(s^{\prime}\right)+\pi \nu\right) \frac{d s^{\prime}}{\rho\left(s^{\prime}\right)} .
$$

We now turn to the effect of a horizontal dipole kick with angle $\theta_{0}$ at location $s_{0}$ on the length of the closed orbit. The position at any location $s$ due to the kick is given by the well-known expression [6]

$$
x(s)=\frac{\sqrt{\beta(s) \beta_{0}}}{2 \sin \pi \nu} \cos \left(\phi(s)-\phi_{0}-\pi \nu\right) \theta_{0}
$$

If the orbit lies further out in bending dipole magnets around the ring we can calculate the change in orbit in a similar fashion how the momentum compaction

$$
\Delta s=\oint \frac{x(s)}{\rho(s)} d s .
$$

$$
\Delta s=\theta_{0} \oint \frac{\sqrt{\beta(s) \beta_{0}}}{2 \sin \pi \nu} \cos \left(\phi(s)-\phi_{0}-\pi \nu\right) \frac{d s}{\rho(s)} .
$$

After using $\cos (x)=\cos (-x)$ we find that the integral equals the expression for the dispersion shown in Eq. 4 and we finally arrive at

$$
\Delta s=\eta_{0} \theta_{0}
$$

which shows that the change of orbit length $\Delta s$ of a dipole kick of magnitude $\theta_{0}$ at 
If there is more than one kick $\theta$ in the ring the total orbit length variation is given as a sum over terms such as that shown in Eq. 8 and we have to sum over all sources of dipole kicks

$$
\Delta s=\sum_{j} \eta_{j} \theta_{j}
$$

where $\theta_{j}$ are the kick angles and $\eta_{j}$ the dispersion at the location labeled $j$ where the kick occurs.

The dipole kick of magnitude $\theta$ from a dipole error labeled $j$ will cause the beam position on a BPM labeled $i$ to change by the response coefficient $C^{i j}$ such that we have $x_{i}=C^{i j} \theta_{j}$. The specific form of the response coefficients in terms of transfer matrices is given in the appendix.

\section{Misalignments}

The primary source of errors are transversely displaced quadrupoles. If they have a focal length $1 / f=k_{1} l$ and are transversely displaced by an amount $\delta \tilde{x}$ the kick they apply to the beam is given by $\theta=\delta \tilde{x} / f$ which causes an orbit length variation $\Delta s^{q}=\tilde{\eta} \delta \tilde{x} / f$ where $\tilde{\eta}$ is the dispersion at the location of the displaced quadrupole. If we consider all misaligned quadrupoles we simply sum over the contributions of all quadrupoles

$$
\Delta s^{q}=\sum_{k} \tilde{\eta}_{k} \frac{\delta \tilde{x}_{k}}{f_{k}}
$$

where the index $k$ labels all quadrupoles. The same displacement will also be visible on BPMs around the ring and is given by the response coefficients $C_{12}^{i j}$ of angle perturbation (subscript 2) at location $j$ and position change (subscript 1) at location $i$ through

$$
x_{i}=\sum_{k} \tilde{C}_{12}^{i k} \frac{\delta \tilde{x}_{k}}{f_{k}}
$$

where $x_{i}$ denotes the change of reading on BPM number $i$ due to the displacement $\delta \tilde{x}_{k}$ of quadrupole number $k$. In the following we will omit the subscripts to make the notation less cluttered. Note that the response coefficients $\tilde{C}^{i k}$ above are treated for thin lens quadrupoles and that we use quantities with a tilde to indicate association with the location of quadrupoles.

A second source of errors are longitudinal displaced dipoles which are equivalent to a small kick of magnitude $\delta \hat{z} / \rho$ at one end and an opposite kick at the other end [7]. The corresponding variation of the orbit length $\Delta s^{d}$ is then given by

$$
\Delta s^{d}=\left(\hat{\eta}_{d}^{\text {out }}-\hat{\eta}_{d}^{\text {in }}\right) \frac{\delta \hat{z}}{\rho}=\frac{\hat{\eta}^{\text {out }}-\hat{\eta}^{\text {in }}}{L} \phi_{d} \delta \hat{z}
$$


where $L, \rho, \phi_{d}=L / \rho$ are the length, bending radius, and the bending angle of the dipole and $\hat{\eta}_{d}$ is the dispersion at either end of the dipole. Summing over all displaced dipoles allows us to write

$$
\Delta s^{d}=\sum_{l} \frac{\hat{\eta}_{l}^{o u t}-\hat{\eta}_{l}^{\text {in }}}{L} \phi_{l} \delta \hat{z}_{l}
$$

where the index $l$ labels the dipole magnets. Note that in the case that the dipoles are very short the quantity $\left(\hat{\eta}_{l}^{\text {out }}-\hat{\eta}_{l}^{\text {in }}\right) / L$ approximately equals the derivative of the dispersion $\hat{\eta}_{l}^{\prime}$ in the dipole.

The displaced dipoles will also affect the transverse position $x_{i}$ of the beam visible on the $\mathrm{BPM}$

$$
x_{i}=\left(\hat{C}_{12}^{i l, \text { out }} \frac{\delta \hat{z}_{l}}{\rho}-\hat{C}_{12}^{i l, \text { in }} \frac{\delta \hat{z}_{l}}{\rho}\right)=\frac{\hat{C}_{12}^{i l, \text { out }}-\hat{C}_{12}^{i l, \text { in }}}{L} \phi_{l} \delta \hat{z}_{l}=\hat{C}^{i j} \phi_{l} \delta \hat{z}_{l}
$$

where $\hat{C}_{12}^{i l, o u t / \text { in }}$ is the response coefficient from the exit and entrance of dipole labeled $l$ to BPM labeled $i$. We introduce the short-hand notation $\hat{C}^{i l}=\left(C_{12}^{i l \text { out }}\right.$ $\left.C_{12}^{i l, i n}\right) / L$ to simplify the notation. Note that if the dipole bends only weakly, the net effect is a transverse displacement of magnitude $-L \delta \hat{z} / \rho=-\phi_{d} \delta \hat{z}$ after the dipole and we have that $\hat{C}^{i l}=-\hat{C}_{11}^{i l}$. Here and in the following we use quantities with a hat to indicate association with the location of dipoles.

The effect of all error sources on the orbit length $\Delta s$ can thus be written as

$$
\Delta s=\Delta s^{q}+\Delta s^{d}=\sum_{k} \tilde{\eta}_{k} \frac{\delta \tilde{x}_{k}}{f_{k}}+\sum_{l} \frac{\hat{\eta}_{l}^{\text {out }}-\hat{\eta}_{l}^{\text {in }}}{L} \phi_{l} \delta \hat{z}_{l}
$$

for transverse quadrupole displacement $\delta \tilde{x}$ and longitudinal dipole displacement $\delta \hat{z}$. Other errors such as excitation errors of the dipole magnets can be included in a similar fashion. Normally these alignment errors lead to a visible orbit distortion, that is corrected with the orbit correction system. The orbit distortion at the BPMs is given by

$$
x_{i}=\sum_{k} \tilde{C}_{12}^{i k} \frac{\delta \tilde{x}_{k}}{f_{k}}+\sum_{l} \hat{C}^{i l} \phi_{l} \delta \hat{z}_{l}
$$

and the correction of that orbit we address in the next section.

\section{Orbit Correction}

The dipole errors discussed so far come from unavoidable imperfections in the construction of the ring which predominantly lead to transverse orbit variations that are visible on BPMs and the BPM readings are subsequently used to calculate excitations for correction steering magnets in order to minimize the observed orbit displacement on the BPMs. Apart from correcting the transverse offset at the 
BPMs the correctors used will also affect the orbit length by virtue of eq. 8 . In this report we use a simple least squares orbit correction system and assume that there are sufficient BPMs available in order to render the orbit correction system non-degenerate. In this case the corrector excitations are determined by

$$
x_{i}=\sum_{j} C_{12}^{i j} \theta_{j}
$$

where $x_{i}$ is the reading of BPM labeled $i$ and $\theta^{j}$ is the excitation of corrector labeled $j$. The response coefficients $C_{12}^{i j}$ between corrector $j$ and BPM $i$ are discussed in the appendix and the sum extends over all corrector magnets. Eq. 17 describes a linear system of equations that can be solved in the least-squares sense by the pseudo-inverse [9]

$$
\theta_{j}=\sum_{i}\left[\left(C^{t} C\right)^{-1} C^{t}\right]_{j i} x_{i}
$$

where we denote the matrix $C_{12}^{i j}$ simply by $C$ and $C^{t}$ denotes the transpose of $C$. The square bracket contains the pseudo-inverse matrix that can be regularized using singular value decomposition, if the inverse should be degenerate or near degenerate.

The final effect we consider is the finite resolution of the BPMs which will cause the orbit correction system to excite the steering magnets with 'wrong' values. The BPM uncertainties from mechanical misalignment, electrical noise or imbalance of the separate channels of a BPM we denote by $\delta x_{i}$.

We now combine the different effects to investigate their respective severity. In order to properly take the orbit correction system into account we write down the effect of all transverse perturbations and the orbit correctors on the BPMs

$$
x_{i}=\delta x_{i}+\sum_{k} \tilde{C}^{i k} \frac{\delta \tilde{x}_{k}}{f_{k}}+\sum_{l} \hat{C}^{i l} \phi_{l} \delta \hat{z}_{l}+\sum_{j} C^{i j} \theta_{j}
$$

where $\delta x_{i}$ are the BPM uncertainties, $\tilde{C}$ and $\hat{C}$ are the response matrices of BPM position to quadrupole offset and longitudinal dipole offset, respectively. $C$ is the response matrix of BPM position to kick angle of the steering correctors.

Orbit correction now works by minimizing the left hand side in the least squares sense with the result

$$
\left.\theta_{j}=-\sum_{i}\left[\left(C^{t} C\right)^{-1} C^{t}\right]\right]_{j i}\left(\delta x_{i}+\sum_{k} \tilde{C}^{i k} \frac{\delta \tilde{x}_{k}}{f_{k}}+\sum_{l} \hat{C}^{i l} \phi_{l} \delta \hat{z}_{l}\right)
$$

and the contribution to the orbit length variation from all corrector magnets is $\Delta s^{c}=\sum_{j} \eta_{j} \theta_{j}$ as already discussed above.

The cumulative effect of misaligned magnets from the previous section and the 
orbit correction on the orbit length is assembled to

$$
\begin{aligned}
\Delta s= & \sum_{k} \tilde{\eta}_{k} \frac{\delta \tilde{x}_{k}}{f_{k}}+\sum_{l} \hat{\eta}_{l}^{\prime} \phi_{l} \delta \hat{z}_{l} \\
& -\sum_{j} \eta_{j} \sum_{i}\left[\left(C^{t} C\right)^{-1} C^{t}\right]_{j i}\left(\delta x_{i}+\sum_{k} \tilde{C}^{i k} \frac{\delta \tilde{x}_{k}}{f_{k}}+\sum_{l} \hat{C}^{i l} \phi_{l} \delta \hat{z}_{l}\right) \\
= & -\sum_{j} \eta_{j} \sum_{i}\left[\left(C^{t} C\right)^{-1} C^{t}\right]_{j i} \delta x_{i} \\
& +\sum_{k}\left[\tilde{\eta}_{k}-\sum_{j} \eta_{j}\left[\left(C^{t} C\right)^{-1} C^{t} \tilde{C}\right]_{j k}\right]_{\frac{\delta}{f_{k}}} \\
& +\sum_{l}\left[\hat{\eta}_{l}^{\prime}-\sum_{j} \eta_{j}\left[\left(C^{t} C\right)^{-1} C^{t} \hat{C}\right]_{j l}\right]_{l} \delta \hat{z}_{l}
\end{aligned}
$$

This equation has a straightforward interpretation. The first term, proportional to $\delta x_{i}$ contains the BPM errors $\delta x_{i}$ that causes the steering correctors to assume a non-zero excitation, which causes the orbit length to change. The second term with the sum over the quadrupole index $k$ contains the single term $\tilde{\eta}_{k}$ which is the direct increase of the orbit length due to the misaligned quadrupole. The second term with $\left(C^{t} C\right)^{-1} C^{t} \tilde{C}$ describes the effect of the quadrupole misalignment on the BPM position through $\tilde{C}$ and then uses the orbit correction matrix $\left(C^{t} C\right)^{-1} C^{t}$ to calculate the steering magnet excitation needed to correct the orbit, which also contributes to the orbit length. The term proportional $\delta \hat{z}_{l}$ has the same interpretation. First the direct orbit length change and second, the corrective effect of the orbit correction system. In short, the factors in the square brackets are the amplification factors of a displacement or BPM errors on the orbit length.

\section{Random Misalignment}

In order to estimate the magnitude of the effect we assume that the misalignments $\delta \tilde{x}_{k}$ and $\delta \hat{z}_{l}$ as well as the BPM error $\delta x_{i}$ are randomly distributed according to Gaussian distributions with mean zero and $\mathrm{rms} \sigma_{Q}, \sigma_{D}$, and $\sigma_{B}$ for quadrupole, dipole and BPM, respectively. In the following we assume that the errors of the different elements are uncorrelated which allows us to consider the BPMs, quadrupoles and dipoles separately.

We start with the BPM resolution and will assume that the BPM errors of different BPMs is uncorrelated and has rms amplitude $\sigma_{B}$ which means $\left\langle\delta x_{i} \delta x_{j}\right\rangle=$ $\sigma_{B}^{2} \delta_{i j}$ where the angle brackets denote ensemble averaging over the BPM error distribution, and $\delta_{i j}$ is the Kronecker delta which is unity if $i=j$ and zero else. 
Calculating the ensemble average of the orbit length $\left\langle\Delta s_{B}^{2}\right\rangle$ we find that

$$
\left\langle\Delta s_{B}^{2}\right\rangle=\sigma_{B}^{2} \sum_{j} \sum_{k} \eta_{j} \eta_{k}\left(C^{t} C\right)_{j k}^{-1}=\alpha_{B}^{2} \sigma_{B}^{2}
$$

where we define the sensitivity of orbit length on the BPM errors as $\alpha_{B}$. Note that the previous expression is the expectation value of the covariance matrix in the state vector of the dispersion at the corrector locations, that can be intuitively written as $\alpha_{B}^{2}=\left\langle\vec{\eta}\left|\left(C^{t} C\right)^{-1}\right| \vec{\eta}\right\rangle$ where we borrow liberally from quantum mechanics for the notation.

For the effect on the orbit length due to quadrupole misalignment we proceed in a similar way and assume that all displacements are uncorrelated. If several magnets are located on the same girder this is not strictly true and can be accounted for by introducing a covariance matrix for the misalignments instead of the Kronecker delta we use by assuming that the ensemble average over different realizations of the misalignments is given by $\left\langle\delta \tilde{x}_{i} \delta \tilde{x}_{j}\right\rangle=\sigma_{Q}^{2} \delta_{i j}$. Evaluating the square of the orbit length variation $\left\langle\Delta s_{Q}^{2}\right\rangle$ we find

$$
\left\langle\Delta s_{Q}^{2}\right\rangle=\sum_{k} \frac{\sigma_{Q}^{2}}{f_{k}^{2}}\left[\tilde{\eta}_{k}-\sum_{j} \eta_{j} F_{j k}\right]\left[\tilde{\eta}_{k}-\sum_{m} \eta_{m} F_{m k}\right]=\alpha_{Q}^{2} \sigma_{Q}^{2}
$$

where we defined the matrix $F=\left(C^{t} C\right)^{-1} C^{t} \tilde{C}$ to simplify the equation and introduced $\alpha_{Q}$, the sensitivity of the orbit length to quadrupole misalignment errors.

For the longitudinal displacement of the dipoles we can do the same analysis, where we first introduce the ensemble average of the longitudinal displacements $\left\langle\delta z_{i} \delta z_{j}\right\rangle=\sigma_{D}^{2} \delta_{i j}$ and for the corresponding orbit length variation $\left\langle\Delta s_{D}^{2}\right\rangle$ we get

$$
\left\langle\Delta s_{D}^{2}\right\rangle=\sum_{l} \phi_{l}^{2} \sigma_{D}^{2}\left[\hat{\eta}_{l}^{\prime}-\sum_{j} \eta_{j} G_{j l}\right]\left[\hat{\eta}_{l}^{\prime}-\sum_{m} \eta_{m} G_{m l}\right]=\alpha_{D}^{2} \sigma_{D}^{2}
$$

where we introduce the abbreviation $G=\left(C^{t} C\right)^{-1} C^{t} \hat{C}$ and the orbit length sensitivity $\alpha_{D}$ in a similar way as before for the BPM and quadrupoles.

\section{FODO}

We tested the method outlined in the previous sections with a ring consisting of 36 FODO cells with an approximate phase advance per cell of 60, 72, 90, and 120 degrees betatron phase advance per cell in both transverse planes and one (sector-) dipole magnet per cell with a deflection angle of 10 degrees. The quadrupole excitations are slightly increased such that the fractional part of the tune for the entire ring was adjusted to $\nu_{x}=0.28$ and $\nu_{y}=0.31$ in order to to 


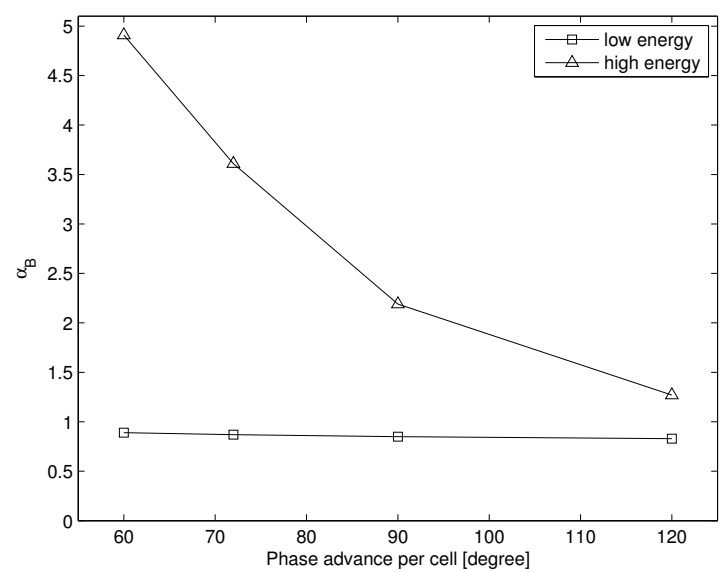

Figure 1: The amplification factors for misalignment of $\mathrm{BPM} \alpha_{B}$ as a function of the phase advance per FODO cell. The lower trace is valid at low energies with $\gamma \rightarrow 0$.

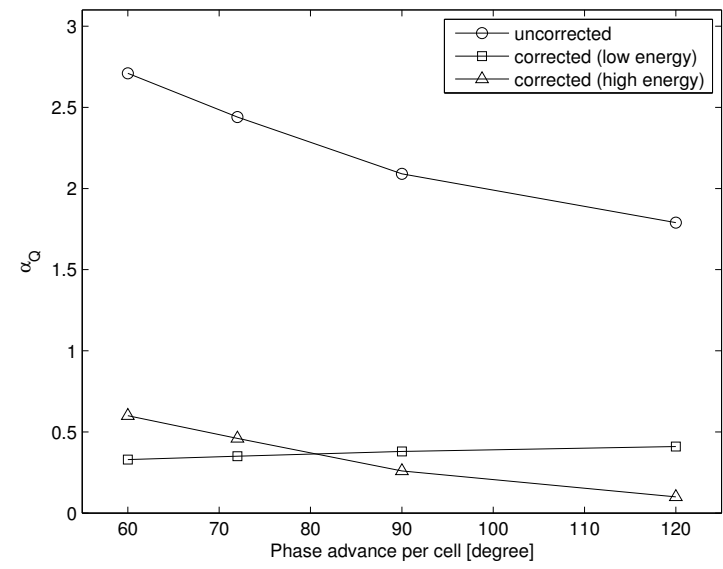

Figure 2: The amplification factors for misalignment of quadrupoles $\alpha_{Q}$ as a function of the phase advance per FODO cell. The upper trace shows the uncorrected factor and the lower traces with correction at low and high energies, respectively.

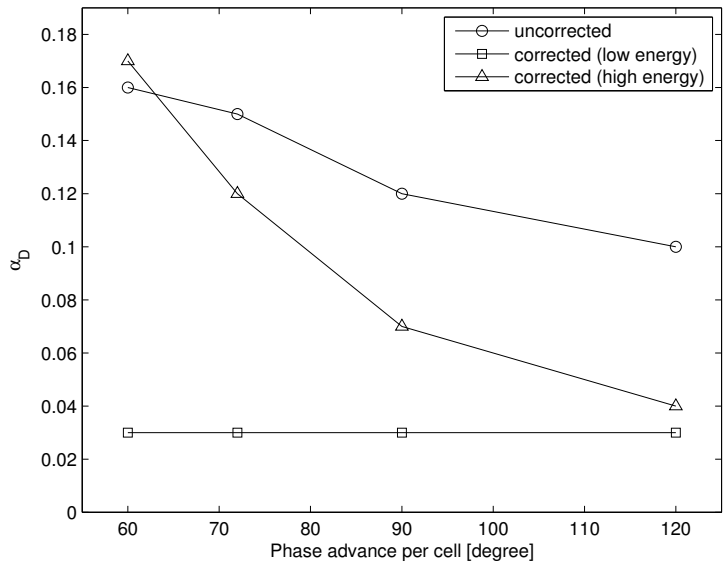

Figure 3: The amplification factors for misalignment of dipoles $\alpha_{D}$ as a function of the phase advance per FODO cell. The upper trace shows the uncorrected factor and the lower traces with correction at low and high energies, respectively. 


\begin{tabular}{l|c|c|c|c} 
Phase advance per cell & $60^{\circ}$ & $72^{\circ}$ & $90^{\circ}$ & $120^{\circ}$ \\
\hline Momentum compaction $\alpha$ & 0.026 & 0.019 & 0.012 & 0.007 \\
\hline BPM $\alpha_{B}$ (low energy) & 0.89 & 0.87 & 0.85 & 0.83 \\
(high energy) & 4.91 & 3.61 & 2.19 & 1.27 \\
\hline Quadrupoles $\alpha_{Q}$ & 2.71 & 2.44 & 2.09 & 1.79 \\
with orbit corr. (low) & 0.33 & 0.35 & 0.38 & 0.41 \\
with orbit corr. (high) & 0.60 & 0.46 & 0.26 & 0.10 \\
\hline Dipoles $\alpha_{D}$ & 0.16 & 0.15 & 0.12 & 0.10 \\
with orbit corr. (low) & 0.03 & 0.03 & 0.03 & 0.03 \\
with orbit corr. (high) & 0.17 & 0.12 & 0.07 & 0.04
\end{tabular}

Table 1: The amplification factors for rings with 36 FODO cells and slightly increased quadrupole excitation to set the fractional tunes to $\nu_{x}=0.28$ and $\nu_{y}=0.31$.

avoid integer tunes. The geometry of the cell is a $2.5 \mathrm{~m}$ long drift space, a thin lens horizontally focusing quadrupole with adjacent BPM and corrector magnet, a drift space of 2, a $1 \mathrm{~m}$ long sector dipole, another $2 \mathrm{~m}$ long drift space, the defocusing quadrupole and the final $2.5 \mathrm{~m}$ long drift section. The circumference of the ring is $360 \mathrm{~m}$ and the momentum compaction factor, which is important for the orbit correction matrix $C$, as can be seen from eq. A.4, is given in table 1. The response coefficients depend on the beam energy through the kinematic factor $\gamma$ in the denominator of the term with the dispersion in eq. A.4 and in the table we display the extreme cases with $\gamma \rightarrow 1$ and $\gamma \rightarrow \infty$. The two cases correspond to a low-energy ion accelerator and an electron accelerator, respectively. Near transition where the denominator becomes singular the response matrix is dominated by the dispersion term and since it is given as the tensor product of the vector containing the dispersion at the correctors and a corresponding vector of the dispersion at the BPM its determinant vanishes and the response matrix is singular. This needs special care and is outside the scope of this report.

We observe in the first row that the momentum compaction factor $\alpha$ decreases with increasing phase advance per cell. The orbit length amplification factor $\alpha_{B}$ for the low-energy rings is below 0.9 and varies only weakly with phase advance as shown in the lower trace on Fig. 1. On the other hand, for the high energy ring, where the dispersion term in the response coefficients is larger, $\alpha_{B}$ is significantly larger as shown in the upper trace in Fig. 1. The situation is worse for lower phase advance rings ( $\alpha_{B}=4.91$ versus 1.27$)$. This behavior can be understood from the fact that the detrimental factor in the response coefficient is proportional to $\eta^{2} / \alpha$ and $\alpha$ is itself proportional to the dispersion such that the extra term in the response matrix is proportional to $\eta$ which gets smaller with increased phase advance per cell.

The upper trace on Fig. 2 shows the quadrupole amplification factor $\alpha_{Q}$ without 


\begin{tabular}{|c|c|c|c|c|}
\hline Configuration & HESR & at $3 \mathrm{GeV}$ & at $8 \mathrm{GeV}$ & at $15 \mathrm{GeV}$ \\
\hline $\operatorname{BPM} \alpha_{B}$ & 0.52 & 0.64 & 1.41 & 3.61 \\
\hline Quadrupoles $\alpha_{Q}$ & 8.68 & & & \\
\hline with orbit corr. & 2.18 & 2.62 & 5.29 & 12.30 \\
\hline $\begin{array}{l}\text { Dipoles } \alpha_{D} \\
\text { with orbit corr. }\end{array}$ & $\begin{array}{l}0.78 \\
0.06\end{array}$ & 0.07 & 0.16 & 0.37 \\
\hline
\end{tabular}

Table 2: The amplification factors for the HESR. The column labeled HESR shows the values ignoring the dispersion term in the response coefficients.

orbit correction varies between 2.71 and 1.79. The lower trace shows that for the low-energy ring the orbit correction reduces the factor to around 0.4 and for the high energy ring in the same order. Fig. 3 shows that the dipole amplification factor $\alpha_{D}$ is below 0.2 for all configurations.

We summarize that all random amplification factors are of order unity where the BPM amplification factors $\alpha_{B}$ for the high energy ring are largest. This implies that the absolute BPM alignment is the most critical in order to guarantee the correct circumference of a ring. But even here the accuracy for the orbit length is given by a few times the absolute BPM positioning accuracy. The other amplification factors for quadrupoles $\alpha_{Q}$ and dipoles $\alpha_{D}$ are smaller to start with and can be reduced below unity by the orbit correction system, such that they will contribute very little to the uncertainty of the FODO rings.

\section{HESR}

The HESR [1] has the shape of a racetrack with a circumference of $574 \mathrm{~m}$. The bending magnets in the arcs with a FODO lattice admit beams with a rigidity of up to $50 \mathrm{Tm}$; for protons the momentum range is $1.5 \mathrm{GeV} / \mathrm{c}$ to $15 \mathrm{GeV} / \mathrm{c}$. One of the long straight sections between the arcs with a FODO structure is occupied by injection elements, the RF system, and the PANDA detector with a Pellet target [8] integrated into the detector. The other long straight section houses an electron cooler and the kickers for the stochastic cooling system.

We performed the orbit length analysis [10] for an early version of the HESR storage ring [11] in the FAIR facility at GSI in Darmstadt, Germany. In that optics the momentum compaction factor was -0.024. For the random orbit length amplification factors we found the values shown in the leftmost column in table 2 labeled 'HESR'. In these simulations we did not take into account the last term with the dispersion in all response matrices. The amplification factors for this configuration without orbit correction are between 0.52 and 8.68 and a rms misalignment tolerance of $0.1 \mathrm{~mm}$ will result in an orbit length uncertainty on the order of $1 \mathrm{~mm}$ for 
the ring with a circumference of $574 \mathrm{~m}$. This amounts to an relative uncertainty of $210^{-6}$. The situation is improved by the orbit correction system where the quadrupole amplification factor is improved by a factor four and the dipole factor even more.

Later we expanded the analysis and included the dispersion term in the response coefficients and repeated the analysis at three different beam energies of $3 \mathrm{GeV}$, $8 \mathrm{GeV}$ and $15 \mathrm{GeV}$. The corresponding orbit amplification factors are reported in the correspondingly labeled columns in table 2. For the BPM amplification factor $\alpha_{B}$ we find that it increases with increasing beam energy, mostly due to the denominator of the dispersion term in the response coefficient, which is largest for small energies which is a behavior we already found for the FODO rings in the previous section. The less important the dispersion term is, the better behaved the response matrix is. Even the other orbit amplification factors $\alpha_{Q}$ and $\alpha_{D}$ increase with increasing energies, approximately in the same proportion as the BPM factor $\alpha_{B}$. It is noteworthy that for the $15 \mathrm{GeV}$ case the orbit correction system makes the quadrupole amplification factor with 12.3 larger than for the uncorrected case, where it is 8.68. Here the orbit correction actually improves the situation for quadrupoles, but the steering magnets themselves affect the orbit length to a larger extent.

We summarize that the orbit amplification factors are on the order of 10 or below and will lead to an orbit length uncertainty of about $1 \mathrm{~mm}$.

\section{Conclusions}

We analyzed the effect of transverse quadrupole misalignment errors, longitudinal dipole positioning errors, and BPM readout errors on the orbit length and quantified the effect by amplification factors of those errors, which we assumed to be randomly distributed and uncorrelated. We considered the case where the effect is uncorrected or corrected by an orbit correction system. We applied the method to a simple ring consisting of FODO cells with phase advance of $60,72,90$, and $120^{\circ}$ and on an early version of the HESR storage ring.

We found that the amplification factors were consistently below about 10 and could be improved by a factor 3 to 10 using the orbit correction system, such that random misalignments of quadrupoles, dipoles and BPMs of $0.1 \mathrm{~mm}$ will result in orbit uncertainties of about $1 \mathrm{~mm}$ or below which is the answer to the question posed in the title of this report. Under most circumstances this implies that the relative orbit uncertainty of the orbit length $\Delta L / L$ is on the order of $10^{-6}$ or below 
which should be sufficient under most circumstances.

It is noteworthy that the orbit correction system and its dependence on the energy through the dispersion term plays a significant role. When writing the orbit response matrix for the orbit correction system the contribution from the dispersion term can be expressed as a tensor product of the vector of the dispersion at the $\mathrm{BPM} \eta_{i}$ and the vector of the dispersion at the corrector magnet $\eta_{j}$. By construction such a matrix is always degenerate, because for example the first column is proportional to $\eta_{i} \eta_{j=1}$ and the second column to $\eta_{i} \eta_{j=2}$. Thus the second column is $\eta_{j=2} / \eta_{j=1}$ times the first column. This holds for any two columns with non-zero $\eta_{j}$ and for $\eta_{j}=0$ the degeneracy is directly obvious. We therefore see that the dispersion contribution of the orbit response matrix is always degenerate and the more important it becomes, for example by a small phase shift factor $\alpha-1 / \gamma^{2}$ the more degenerate the full orbit response matrix becomes. This behavior lies behind the increasing orbit amplification factors for larger energies. On the other hand, the dispersion term is minimum for storage rings operating at low energies, such a low-energy ion rings, where $\gamma$ is close to unity and the term $\alpha-1 / \gamma^{2} \approx-1$.

[1] R. Maier, et al., The High-energy storage ring (HESR), Proceedings of the 24th Particle Accelerator Conference in Washington, D.C., (2011) 2104.

[2] W. Henning (ed.), An international Accelerator Facility for Beams of Ions and Antiprotons, Conceptual Design Report, GSI, Darmstadt, November 2001.

[3] PANDA Collaboration: W. Erni, et al., Physics Performance Report for PANDA: Strong Interaction Studies with Antiprotons, arXiv:0903.3905 [hepex] (2009).

[4] S. Holm, et. al., New Accelerators in Uppsala, Physica Scripta 34 (1986) 513.

[5] S. Häggström, Production of $\eta$-mesons in Proton-Neutron Collisions, Uppsala Dissertations from the Faculty of Science and Technology 13, 1997.

[6] H. Wiedemann, Particle Accelerator Physics, 2nd ed., Springer Verlag, Berlin, 2003.

[7] B. Brown, Closed orbit effects due to longitudinal bend center displacement, Fermilab, MI-0162 (1996), unpublished.

[8] B. Trostell, Vacuum injection of hydrogen microsphere beams, Nuclear Instruments and Methods A 362 (1995) 41. 
[9] W. Press, et al., Numerical Recipes, Cambridge University Press, Cambridge, 1986.

[10] M. Beckmann, Length Variation of Beam Orbits in Circular Accelerators, Uppsala University, June 2007, unpublished.

[11] D. Welsch, Auslegung einer Orbitkorrektursystems für den Hochenergie Speicherring HESR im Projekt FAIR, Berichte des Forschungszentrums Jülich; Jül-4241, 2007.

\section{Appendix A. Response Coefficients}

The effect of a single dipole kick at location labeled $j$ in a storage ring will cause an orbit displacement $x_{j}$ immediately after the kick, that is described by

$$
\vec{x}_{j}=R^{j j} \vec{x}_{j}+\vec{\theta}_{j}
$$

where we denote the $4 \times 4$ position vector $\left(x \cdot x^{\prime}, y, y^{\prime}\right)$ by $\vec{x}$, and $R^{j j}$ is the $4 \times 4$ transfer matrix for a full turn starting immediately after the kick. The kick $\vec{\theta}$ is the vectorized form of the kick vector $(0, \theta, 0,0)$ where only the horizontal angle $x^{\prime}$ is changed. Solving the previous equation yields the closed orbit change as

$$
\vec{x}_{j}=\left(1-R^{j j}\right)^{-1} \vec{\theta}_{j}
$$

and the orbit change at the BPM labeled $i$ is given by

$$
\vec{x}_{i}=R^{i j} \vec{x}_{j}=R^{i j}\left(1-R^{j j}\right)^{-1} \vec{\theta}_{j}
$$

where $R^{i j}$ is the transfer matrix from the dipole kick at location labeled $j$ to the BPM at location labeled $i$.

We need, however, also consider that the dipole kick changes the orbit length by an amount $\eta_{j} \theta$ according to eq. 8. The discussion so far did not consider that the revolution frequency is maintained fixed due to the presence of a RF system. This constraint causes the beam to move to a different $\mathrm{RF}$ phase in order to change its energy to maintain the revolution frequency constant, despite the lengthened orbit. This change of beam momentum is given by $\Delta p / p=-\eta_{j} \theta /\left(\alpha-1 / \gamma^{2}\right) C$ from eq. 2 . For a single steering magnet this will cause the transverse beam position, visible on the BPMs, to change by an additional amount $\eta_{i} \Delta p / p$, thus we get for the full response coefficient

$$
C_{12}^{i j}=\left[R^{i j}\left(1-R^{j j}\right)^{-1}\right]_{12}-\frac{\eta_{i} \eta_{j}}{\left(\alpha-1 / \gamma^{2}\right) C}
$$

where the first term is due to the normal position change and the second term due to the adapting energy to maintain a constant revolution frequency. 